\title{
A NEW TWO-URN MODEL
}

\author{
MAY-RU CHEN, ${ }^{*}$ National Sun Yat-sen University \\ SHOOU-REN HSIAU ***** AND \\ TING-HSIN YANG, ${ }^{* *}$ National Changhua University of Education
}

\begin{abstract}
We propose a two-urn model of Pólya type as follows. There are two urns, urn $A$ and urn $B$. At the beginning, urn $A$ contains $r_{A}$ red and $w_{A}$ white balls and urn $B$ contains $r_{B}$ red and $w_{B}$ white balls. We first draw $m$ balls from urn $A$ and note their colors, say $i$ red and $m-i$ white balls. The balls are returned to urn $A$ and $b i$ red and $b(m-i)$ white balls are added to urn $B$. Next, we draw $\ell$ balls from urn $B$ and note their colors, say $j$ red and $\ell-j$ white balls. The balls are returned to urn $B$ and $a j$ red and $a(\ell-j)$ white balls are added to urn $A$. Repeat the above action $n$ times and let $X_{n}$ be the fraction of red balls in urn $A$ and $Y_{n}$ the fraction of red balls in urn $B$. We first show that the expectations of $X_{n}$ and $Y_{n}$ have the same limit, and then use martingale theory to show that $X_{n}$ and $Y_{n}$ converge almost surely to the same limit.
\end{abstract}

Keywords: Two-urn model; martingale

2010 Mathematics Subject Classification: Primary 60F15

Secondary 60G42; 60E05

\section{Introduction}

The study of urn models has a long history. James Bernoulli (1713) may be the first person to mention problems in the language of urns (cf. [10]). Up to 1977, the results on urn models were summarized in the book 'Urn Models and Their Applications' [9]. This book stimulated many probabilists and statisticians to investigate different kinds of urn models. After two decades, Kotz and Balakrishnan [10] published a survey paper: 'Advances in Urn Models During the Past Two Decades'. It covered almost all of the different kinds of urn models and their properties. One of the most famous urn models was introduced by Eggenberger and Pólya [5], and is usually called the Pólya urn; it is described as follows. An urn initially contains $r$ red and $w$ white balls. A ball is drawn at random and then replaced together with $c$ balls of the same color. The procedure is repeated ad infinitum. It is well-known that the fraction of red balls converges almost surely to a beta distributed random variable with parameters $r / c$ and $w / c$.

Various generalizations of the Pólya urn can be found in the literature. Hill et al. [8] and Higueras et al. [7] extended the result of Eggenberger and Pólya to an urn model in which the probability of adding balls is a function of the fraction of red balls. Bagchi and Pal [2] considered a generalization of the Pólya urn model in which the rule for adding balls follows a matrix. Subsequently, Gouet [6] showed that if the matrix satisfies some suitable conditions,

Received 01 June 2012; revision received 11 January 2013.

* Postal address: Department of Applied Mathematics, National Sun Yat-sen University, 70 Lien-hai Rd., Kaohsiung 804, Taiwan, R. O. C. Email address: mayru@faculty.nsysu.edu.tw

** Postal address: Department of Mathematics, National Changhua University of Education, No. 1 Jin-De Road, Changhua 500, Taiwan, R. O. C.

*** Email address: srhsiau@cc.ncue.edu.tw 
the limit of the fraction of red balls degenerates to a constant. The generalization of the Pólya urn given by Pemantle [13] is a time-dependent urn model. Chen and Wei [4] used martingales to study a generalization of the Pólya urn in which at least two balls are drawn at each time step. Later, Renlund [14] studied a generalized Pólya urn (similar to Chen and Wei's) via stochastic approximation. Recently, Mahmoud [11] wrote a book named 'Pólya urn model', which includes several classical urn models and presents different techniques to investigate various structural properties of Eggenberger-Pólya urn schemes.

One famous generalization of the Pólya urn is the Ehrenfest urn model, which is a two-urn model and may be described as follows. There are two urns, say $A$ and $B$, and $N$ balls, numbered consecutively from 1 to $N$, distributed in the two urns. At each step, an integer between 1 and $N$ is chosen at random, uniformly, and the ball whose number has been chosen is moved to the opposite urn. The procedure is repeated $n$ times and the probability distribution of the number of balls in each urn is considered. Much of the literature on the Ehrenfest urn model is surveyed in [10]. Some of the literature (e.g. [3] and [12]) studied other two-urn models.

In this paper we introduce a new two-urn model, which is described as follows. Assume that there are two urns, urn $A$ and urn $B$. Suppose that, at the beginning, there are $s_{0} x_{0}$ red and $s_{0}\left(1-x_{0}\right)$ white balls in urn $A$, and $t_{0} y_{0}$ red and $t_{0}\left(1-y_{0}\right)$ white balls in urn $B$. Note that $s_{0}$ and $t_{0}$ are the respective number of balls in urns $A$ and $B$, and $x_{0}$ and $y_{0}$ are the respective fraction of red balls in urns $A$ and $B$. First, draw $m \leq s_{0}$ balls (without replacement) from urn $A$ and note their colors, say $i$ red and $m-i$ white balls. Then return the balls to urn $A$ and add $b i$ red and $b(m-i)$ white balls to urn $B$. Next, draw $\ell \leq t_{0}+b m$ balls (without replacement) from urn $B$ and note their colors, say $j$ red balls and $\ell-j$ white balls. Then return the balls to urn $B$ and add $a j$ red and $a(\ell-j)$ white balls to urn $A$. Repeat the above action ad infinitum.

After the $n$th action, let $R_{A}(n)$ and $R_{B}(n)$ be the respective numbers of red balls in the urns $A$ and $B$. At this stage, there are $s_{0}+a \ell n$ balls in urn $A$ and $t_{0}+b m n$ balls in urn $B$. Denote $s_{0}+a \ell n=s_{n}$ and $t_{0}+b m n=t_{n}$ for $n \geq 1$. Let $X_{n}=R_{A}(n) / s_{n}$ and $Y_{n}=R_{B}(n) / t_{n}$, that is, $X_{n}$ and $Y_{n}$ are the respective fractions of red balls in the urns $A$ and $B$ after the $n$th action. Let $\mathcal{F}_{n}$ denote the $\sigma$-field generated by $X_{1}, X_{2}, \ldots, X_{n}$ and $Y_{1}, Y_{2}, \ldots, Y_{n}$. Then $\left(R_{A}(n), R_{B}(n)\right)$ is clearly a Markov chain. For clarity, let

$$
R_{A}(n+1)=R_{A}(n)+a \ell U_{n+1}, \quad R_{B}(n+1)=R_{B}(n)+b m V_{n+1},
$$

where $m V_{n}$ and $\ell U_{n}$ are the respective numbers of red balls drawn from the urns $A$ and $B$ in the $n$th action. Then

$$
\begin{gathered}
\mathbb{P}\left(V_{n+1}=\frac{i}{m} \mid \mathcal{F}_{n}\right)=\frac{\left(\begin{array}{c}
s_{n} X_{n} \\
i
\end{array}\right)\left(\begin{array}{c}
s_{n}-s_{n} X_{n} \\
m-i
\end{array}\right)}{\left(\begin{array}{c}
s_{n} \\
m
\end{array}\right)}, \\
\mathbb{P}\left(U_{n+1}=\frac{j}{\ell} \mid \mathcal{F}_{n}, V_{n+1}\right)=\frac{\left(\begin{array}{c}
t_{n+1} Y_{n+1} \\
j
\end{array}\right)\left(\begin{array}{c}
t_{n+1}-t_{n+1} Y_{n+1} \\
\ell-j
\end{array}\right)}{\left(\begin{array}{c}
t_{n+1} \\
\ell
\end{array}\right)},
\end{gathered}
$$

giving

$$
\mathbb{P}\left(U_{n+1}=\frac{j}{\ell} \mid \mathcal{F}_{n}\right)=\sum_{i=0}^{m} \frac{\left(\begin{array}{c}
t_{n} Y_{n}+b i \\
j
\end{array}\right)\left(\begin{array}{c}
t_{n+1}-t_{n} Y_{n}-b i \\
\ell-j
\end{array}\right)}{\left(\begin{array}{c}
t_{n+1} \\
\ell
\end{array}\right)} \frac{\left(\begin{array}{c}
s_{n} X_{n} \\
i
\end{array}\right)\left(\begin{array}{c}
s_{n}-s_{n} X_{n} \\
m-i
\end{array}\right)}{\left(\begin{array}{c}
s_{n} \\
m
\end{array}\right)} .
$$


Moreover, we have, for $n \geq 0$,

$$
\begin{gathered}
X_{n+1}=\frac{R_{A}(n+1)}{s_{n+1}}=\frac{s_{n} X_{n}+a \ell U_{n+1}}{s_{n+1}}=\alpha_{n} X_{n}+\bar{\alpha}_{n} U_{n+1}=X_{n}+\bar{\alpha}_{n}\left(U_{n+1}-X_{n}\right), \\
Y_{n+1}=\frac{R_{B}(n+1)}{t_{n+1}}=\frac{t_{n} Y_{n}+b m V_{n+1}}{t_{n+1}}=\beta_{n} Y_{n}+\bar{\beta}_{n} V_{n+1}=Y_{n}+\bar{\beta}_{n}\left(V_{n+1}-Y_{n}\right),
\end{gathered}
$$

where $\alpha_{n}=s_{n} / s_{n+1}=1-\bar{\alpha}_{n}$ and $\beta_{n}=t_{n} / t_{n+1}=1-\bar{\beta}_{n}$.

From the above conditional distributions of $V_{n+1}$, it follows that $m V_{n+1}$ is a (conditional) hypergeometric random variable with parameters $s_{n}, s_{n} X_{n}$, and $m$. Hence,

$$
\mathbb{E}\left(V_{n+1} \mid \mathcal{F}_{n}\right)=\frac{1}{m} \mathbb{E}\left(m V_{n+1} \mid \mathcal{F}_{n}\right)=\frac{1}{m} \frac{m s_{n} X_{n}}{s_{n}}=X_{n} .
$$

Similarly, since $\ell U_{n+1}$ is a conditional hypergeometric random variable with parameters $t_{n+1}$, $t_{n+1} Y_{n+1}$, and $\ell$, given $\mathcal{F}_{n}$ and $V_{n+1}$, we have

$$
\mathbb{E}\left(U_{n+1} \mid \mathcal{F}_{n}, V_{n+1}\right)=\frac{1}{\ell} \mathbb{E}\left(\ell U_{n+1} \mid \mathcal{F}_{n}, V_{n+1}\right)=\frac{1}{\ell} \frac{\ell t_{n+1} Y_{n+1}}{t_{n+1}}=\beta_{n} Y_{n}+\bar{\beta}_{n} V_{n+1} .
$$

Thus,

$$
\begin{aligned}
\mathbb{E}\left(U_{n+1} \mid \mathcal{F}_{n}\right) & =\mathbb{E}\left(\mathbb{E}\left(U_{n+1} \mid \mathcal{F}_{n}, V_{n+1}\right) \mid \mathcal{F}_{n}\right) \\
& =\mathbb{E}\left(\beta_{n} Y_{n}+\bar{\beta}_{n} V_{n+1} \mid \mathcal{F}_{n}\right) \\
& =\beta_{n} Y_{n}+\bar{\beta}_{n} X_{n} .
\end{aligned}
$$

Combining identities (1.1)-(1.4) yields

$$
\begin{gathered}
\mathbb{E}\left(X_{n+1} \mid \mathcal{F}_{n}\right)=X_{n}-\bar{\alpha}_{n} \beta_{n}\left(X_{n}-Y_{n}\right), \\
\mathbb{E}\left(Y_{n+1} \mid \mathcal{F}_{n}\right)=Y_{n}+\bar{\beta}_{n}\left(X_{n}-Y_{n}\right) .
\end{gathered}
$$

From (1.5) and (1.6), it is easy to derive

$$
\mathbb{E}\left(X_{n+1}-Y_{n+1}\right)=\alpha_{n} \beta_{n} \mathbb{E}\left(X_{n}-Y_{n}\right)=\cdots=\left(x_{0}-y_{0}\right) \prod_{i=0}^{n} \alpha_{i} \beta_{i}=\frac{s_{0} t_{0}\left(x_{0}-y_{0}\right)}{s_{n+1} t_{n+1}},
$$

and, hence, we have $\lim _{n \rightarrow \infty} \mathbb{E}\left(X_{n}-Y_{n}\right)=0$ since $\lim _{n \rightarrow \infty} 1 /\left(s_{n+1} t_{n+1}\right)=0$.

Motivated by the above result, it is natural to consider the limit of $\mathbb{E} X_{n}$. In Section 2 we first derive the limit of $\mathbb{E} X_{n}$ and this implies that $\mathbb{E} X_{n}$ and $\mathbb{E} Y_{n}$ have the same limit since $\lim _{n \rightarrow \infty} \mathbb{E}\left(X_{n}-Y_{n}\right)=0$. In Section 3 we strengthen the result to the strong convergence; we prove that $X_{n}$ and $Y_{n}$ converge almost surely to the same limit. This is a more involved work. In fact we can use martingale theory to prove that $X_{n}+Y_{n}$ converges almost surely and $X_{n}-Y_{n}$ converges to 0 almost surely; these two results then imply the claim.

\section{Limits of $\mathbb{E} X_{n}$ and $\mathbb{E} Y_{n}$}

In this section we study the limits of $\mathbb{E} X_{n}$ and $\mathbb{E} Y_{n}$. Taking the expectation of both sides of (1.5) and (1.6), and using (1.7) repeatedly, we obtain

$$
\mathbb{E} X_{n}=x_{0}-\sum_{j=0}^{n-1} \frac{a \ell s_{0} t_{0}\left(x_{0}-y_{0}\right)}{s_{j} s_{j+1} t_{j+1}} \quad \text { and } \mathbb{E} Y_{n}=y_{0}+\sum_{j=0}^{n-1} \frac{b m s_{0} t_{0}\left(x_{0}-y_{0}\right)}{s_{j} t_{j} t_{j+1}} .
$$


Note that

$$
\sum_{j=0}^{\infty} \frac{1}{s_{j} s_{j+1} t_{j+1}} \leq \sum_{j=1}^{\infty} \frac{1}{j^{3}}<\infty \quad \text { and } \quad \sum_{j=0}^{\infty} \frac{1}{s_{j} t_{j} t_{j+1}} \leq \sum_{j=1}^{\infty} \frac{1}{j^{3}}<\infty .
$$

Thus, by (2.1), we obtain

$$
\lim _{n \rightarrow \infty} \mathbb{E} X_{n}=x_{0}-\sum_{j=0}^{\infty} \frac{a \ell s_{0} t_{0}\left(x_{0}-y_{0}\right)}{s_{j} s_{j+1} t_{j+1}} \quad \text { and } \lim _{n \rightarrow \infty} \mathbb{E} Y_{n}=y_{0}+\sum_{j=0}^{\infty} \frac{b m s_{0} t_{0}\left(x_{0}-y_{0}\right)}{s_{j} t_{j} t_{j+1}}
$$

Moreover, since $\lim _{n \rightarrow \infty} \mathbb{E}\left(X_{n}-Y_{n}\right)=0$, we have

$$
\lim _{n \rightarrow \infty} \mathbb{E} X_{n}=\lim _{n \rightarrow \infty} \mathbb{E} Y_{n}
$$

It is interesting to note that if $x_{0}=y_{0}$ then, in view of (2.1), $\mathbb{E} X_{n}=\mathbb{E} Y_{n}=x_{0}$ for each $n$, and so $\lim _{n \rightarrow \infty} \mathbb{E} X_{n}=\lim _{n \rightarrow \infty} \mathbb{E} Y_{n}=x_{0}$. In the case $x_{0} \neq y_{0}$ we find a simpler form of $\lim _{n \rightarrow \infty} \mathbb{E} X_{n}$ under the assumptions $a \ell=b m$ and $s_{0}=t_{0}+k a \ell, k \in \mathbb{Z}$.

Theorem 2.1. In the two-urn model, assume that $x_{0} \neq y_{0}$, al $=b m$, and $s_{0}=t_{0}+k a \ell$ for $k \in \mathbb{Z}$. Then

$$
\lim _{n \rightarrow \infty} \mathbb{E} X_{n}= \begin{cases}y_{0}-\left(x_{0}-y_{0}\right)\left(\frac{s_{0}}{a \ell k}-\frac{s_{0} t_{0}}{a \ell k(k+1)} \sum_{j=0}^{k} \frac{1}{s_{0}+a \ell j}\right) & \text { if } k \leq-2, \\ \frac{1}{2}\left(x_{0}+y_{0}\right) & \text { if } k=-1, \\ y_{0}+\left(x_{0}-y_{0}\right)\left(\sum_{j=0}^{\infty} \frac{s_{0}^{2}}{\left(s_{0}+a \ell j\right)^{2}}-\frac{s_{0}}{a \ell}\right) & \text { if } k=0, \\ x_{0}+\left(x_{0}-y_{0}\right)\left(\frac{t_{0}}{a \ell}-\sum_{j=1}^{\infty} \frac{s_{0} t_{0}}{\left(t_{0}+a \ell j\right)^{2}}\right) & \text { if } k=1, \\ a \ell k(k-1) & \text { if } k \geq 2 .\end{cases}
$$

In particular, if $x_{0}=1, y_{0}=0, s_{0}=t_{0}=1$, and $a=b=\ell=m=1$ then we have $\lim _{n \rightarrow \infty} \mathbb{E} X_{n}=\pi^{2} / 6-1$.

Proof. Since $\lim _{n \rightarrow \infty} \mathbb{E} X_{n}=\lim _{n \rightarrow \infty} \mathbb{E} Y_{n}$ we have

$$
\lim _{n \rightarrow \infty} \mathbb{E} X_{n}=\frac{1}{2} \lim _{n \rightarrow \infty} \mathbb{E}\left(X_{n}+Y_{n}\right)
$$

By the assumption $a \ell=b m$ and (2.1), it follows that

$$
\mathbb{E}\left(X_{n}+Y_{n}\right)=x_{0}+y_{0}+s_{0} t_{0}\left(x_{0}-y_{0}\right) \sum_{j=0}^{n-1} \frac{a \ell}{s_{j} t_{j+1}}\left(\frac{1}{t_{j}}-\frac{1}{s_{j+1}}\right) .
$$

If $s_{0}=t_{0}-a \ell$ then $s_{j+1}=t_{j}$ for all $j$ and 2.2 gives $\mathbb{E}\left(X_{n}+Y_{n}\right)=x_{0}+y_{0}$. This implies that

$$
\lim _{n \rightarrow \infty} \mathbb{E} X_{n}=\frac{1}{2}\left(x_{0}+y_{0}\right)
$$


If $s_{0}=t_{0}$ then $s_{j}=t_{j}$ for all $j$ and so, from (2.2),

$$
\begin{aligned}
\mathbb{E}\left(X_{n}+Y_{n}\right) & =x_{0}+y_{0}+s_{0}^{2}\left(x_{0}-y_{0}\right) \sum_{j=0}^{n-1}\left(\frac{1}{s_{j}}-\frac{1}{s_{j+1}}\right)^{2} \\
& =x_{0}+y_{0}+s_{0}^{2}\left(x_{0}-y_{0}\right) \sum_{j=0}^{n-1}\left[\frac{2}{a \ell}\left(\frac{1}{s_{j+1}}-\frac{1}{s_{j}}\right)+\frac{1}{s_{j}^{2}}+\frac{1}{s_{j+1}^{2}}\right] \\
& =x_{0}+y_{0}+s_{0}^{2}\left(x_{0}-y_{0}\right)\left[\frac{2}{a \ell}\left(\frac{1}{s_{n}}-\frac{1}{s_{0}}\right)-\frac{1}{s_{0}^{2}}-\frac{1}{s_{n}^{2}}+\sum_{j=0}^{n} \frac{2}{s_{j}^{2}}\right] \\
& =x_{0}+y_{0}+\left(x_{0}-y_{0}\right)\left[\frac{2 s_{0}^{2}}{a \ell}\left(\frac{1}{s_{n}}-\frac{1}{s_{0}}\right)-1-\frac{s_{0}^{2}}{s_{n}^{2}}+\sum_{j=0}^{n} \frac{2 s_{0}^{2}}{s_{j}^{2}}\right]
\end{aligned}
$$

Thus,

$$
\begin{aligned}
\lim _{n \rightarrow \infty} \mathbb{E} X_{n} & =\frac{1}{2}\left\{x_{0}+y_{0}+\left(x_{0}-y_{0}\right)\left[-\frac{2 s_{0}}{a \ell}-1+\sum_{j=0}^{\infty} \frac{2 s_{0}^{2}}{s_{j}^{2}}\right]\right\} \\
& =y_{0}+\left(x_{0}-y_{0}\right)\left[\sum_{j=0}^{\infty} \frac{s_{0}^{2}}{\left(s_{0}+a \ell j\right)^{2}}-\frac{s_{0}}{a \ell}\right] .
\end{aligned}
$$

If $s_{0}=t_{0}+k a \ell, k \geq 2$, then $s_{j}=t_{j+k}$ for all $j$ and so, from (2.2),

$$
\mathbb{E}\left(X_{n}+Y_{n}\right)
$$

$$
\begin{gathered}
=x_{0}+y_{0}+s_{0} t_{0}\left(x_{0}-y_{0}\right) \sum_{j=0}^{n-1} \frac{(k+1) a^{2} \ell^{2}}{t_{j} t_{j+1} t_{j+k} t_{j+k+1}} \\
=x_{0}+y_{0}+s_{0} t_{0}\left(x_{0}-y_{0}\right) \sum_{j=0}^{n-1}\left[\frac{1}{a \ell k}\left(\frac{1}{t_{j}}-\frac{1}{t_{j+k+1}}\right)-\frac{k+1}{a \ell k(k-1)}\left(\frac{1}{t_{j+1}}-\frac{1}{t_{j+k}}\right)\right] \\
=x_{0}+y_{0}+\left(x_{0}-y_{0}\right)\left[\frac{s_{0} t_{0}}{a \ell k}\left(\frac{1}{t_{0}}+\frac{1}{s_{0}}-\frac{1}{t_{n}}-\frac{1}{t_{n+k}}\right)\right. \\
\left.-\frac{2 s_{0} t_{0}}{a \ell k(k-1)}\left(\sum_{j=1}^{k-1} \frac{1}{t_{j}}-\sum_{j=n+1}^{n+k-1} \frac{1}{t_{j}}\right)\right] .
\end{gathered}
$$

Thus,

$$
\begin{aligned}
\lim _{n \rightarrow \infty} \mathbb{E} X_{n} & =\frac{1}{2}\left\{x_{0}+y_{0}+\left(x_{0}-y_{0}\right)\left[\frac{s_{0}+t_{0}}{a \ell k}-\frac{2 s_{0} t_{0}}{a \ell k(k-1)} \sum_{j=1}^{k-1} \frac{1}{t_{j}}\right]\right\} \\
& =x_{0}+\left(x_{0}-y_{0}\right)\left(\frac{t_{0}}{a \ell k}-\frac{s_{0} t_{0}}{a \ell k(k-1)} \sum_{j=1}^{k-1} \frac{1}{t_{j}}\right) \\
& =x_{0}+\left(x_{0}-y_{0}\right)\left(\frac{t_{0}}{a \ell k}-\frac{s_{0} t_{0}}{a \ell k(k-1)} \sum_{j=1}^{k-1} \frac{1}{t_{0}+a \ell j}\right),
\end{aligned}
$$

where the second equality follows from $s_{0}=t_{0}+k a \ell$. Because the proofs for $k=1$ and $k \leq-2$ are similar to the proofs for $k=0$ and $k \geq 2$, respectively, we omit them here. 


\section{Strong convergence of $X_{n}$}

In this section we study the long term behavior of the processes $\left\{X_{n}\right\}_{n \geq 1}$ and $\left\{Y_{n}\right\}_{n \geq 1}$. Because $\mathbb{E} X_{n}$ and $\mathbb{E} Y_{n}$ converge to the same limit, we are motivated to prove that $X_{n}$ and $Y_{n}$ converge almost surely to the same limit. To this end, we construct a martingale and a supermartingale in terms of $X_{n}+Y_{n}$ and $X_{n}-Y_{n}$.

Theorem 3.1. In the two-urn model,

$$
\left\{X_{n}+Y_{n}+\sum_{k=0}^{n-1}\left(\bar{\alpha}_{k} \beta_{k}-\bar{\beta}_{k}\right)\left(X_{k}-Y_{k}\right)\right\}_{n \geq 1}
$$

is a bounded martingale and $X_{n}+Y_{n}$ converges almost surely; moreover, $\left\{\left(X_{n}-Y_{n}\right)^{2}+4 / n\right\}_{n \geq 1}$ is a nonnegative supermartingale.

Proof. From (1.5) and (1.6), we have, for any $\ell, m \geq 1$,

$$
\mathbb{E}\left[X_{n+1}+Y_{n+1} \mid \mathcal{F}_{n}\right]=X_{n}+Y_{n}-\left(\bar{\alpha}_{n} \beta_{n}-\bar{\beta}_{n}\right)\left(X_{n}-Y_{n}\right),
$$

so $\left\{X_{n}+Y_{n}+\sum_{k=0}^{n-1}\left(\bar{\alpha}_{k} \beta_{k}-\bar{\beta}_{k}\right)\left(X_{k}-Y_{k}\right)\right\}_{n \geq 1}$ is a martingale. Furthermore, since $\bar{\alpha}_{n} \beta_{n}-$ $\bar{\beta}_{n}=O\left(n^{-2}\right)$, the compensator

$$
\sum_{k=0}^{n-1}\left(\bar{\alpha}_{k} \beta_{k}-\bar{\beta}_{k}\right)\left(X_{k}-Y_{k}\right)
$$

converges almost surely. This implies that the martingale (3.1) is bounded and, therefore, convergent almost surely. Hence, $X_{n}+Y_{n}$ converges almost surely.

Next, from (1.1) and (1.2), it follows that

$$
\begin{aligned}
\left(X_{n+1}-\right. & \left.Y_{n+1}\right)^{2} \\
= & \left(X_{n}-Y_{n}\right)^{2}+\left[\bar{\alpha}_{n}\left(U_{n+1}-X_{n}\right)-\bar{\beta}_{n}\left(V_{n+1}-Y_{n}\right)\right]^{2} \\
& \quad+2\left(X_{n}-Y_{n}\right)\left[\bar{\alpha}_{n}\left(U_{n+1}-X_{n}\right)-\bar{\beta}_{n}\left(V_{n+1}-Y_{n}\right)\right] \\
\leq & \left(X_{n}-Y_{n}\right)^{2}+\frac{4}{(n+1)^{2}}+2\left(X_{n}-Y_{n}\right)\left[\bar{\alpha}_{n}\left(U_{n+1}-X_{n}\right)-\bar{\beta}_{n}\left(V_{n+1}-Y_{n}\right)\right],
\end{aligned}
$$

where the last inequality holds since $0<\bar{\alpha}_{n}, \bar{\beta}_{n} \leq 1 /(n+1)$ and $0 \leq X_{n}, Y_{n}, U_{n+1}, V_{n+1} \leq 1$. This together with (1.3) and (1.4) implies that

$$
\begin{aligned}
& \mathbb{E}\left[\left(X_{n+1}-Y_{n+1}\right)^{2} \mid \mathcal{F}_{n}\right] \\
& \quad \leq\left(X_{n}-Y_{n}\right)^{2}+\frac{4}{(n+1)^{2}}+2\left(X_{n}-Y_{n}\right) \mathbb{E}\left[\bar{\alpha}_{n}\left(U_{n+1}-X_{n}\right)-\bar{\beta}_{n}\left(V_{n+1}-Y_{n}\right) \mid \mathcal{F}_{n}\right] \\
& \quad=\left(1-2 \bar{\alpha}_{n} \beta_{n}-2 \bar{\beta}_{n}\right)\left(X_{n}-Y_{n}\right)^{2}+\frac{4}{(n+1)^{2}} .
\end{aligned}
$$

Since $1-2 \bar{\alpha}_{n} \beta_{n}-2 \bar{\beta}_{n}<1$, we have, from (3.2),

$$
\mathbb{E}\left[\left(X_{n+1}-Y_{n+1}\right)^{2}+\frac{4}{n+1} \mid \mathcal{F}_{n}\right] \leq\left(X_{n}-Y_{n}\right)^{2}+\frac{4}{(n+1)^{2}}+\frac{4}{n+1} \leq\left(X_{n}-Y_{n}\right)^{2}+\frac{4}{n} .
$$

Hence, $\left\{\left(X_{n}-Y_{n}\right)^{2}+4 / n\right\}_{n \geq 1}$ is a nonnegative supermartingale. 
We are now ready to prove our main result.

Theorem 3.2. In the two-urn model, the fractions of red balls in urns A and B converge almost surely to the same limit.

Proof. In view of Theorem 3.1, $X_{n}+Y_{n}$ converges almost surely. If we can further prove that $X_{n}-Y_{n}$ converges to 0 almost surely, then the proof is complete. Again Theorem 3.1 and the supermartingale convergence theorem (see [1, p. 419]) imply that $\left(X_{n}-Y_{n}\right)^{2}+4 / n$ converges almost surely and so does $\left(X_{n}-Y_{n}\right)^{2}$ since $4 / n$ converges to 0 . Let $Z_{n}=\left(X_{n}-Y_{n}\right)^{2}$ and $Z_{n} \rightarrow Z$ almost surely. We claim that $\mathbb{E} Z_{n} \rightarrow 0$. If so, then, by the dominated convergence theorem, $\mathbb{E} Z=0$ and, therefore, $Z=0$ almost surely. This implies that $X_{n}-Y_{n}$ converges to 0 almost surely. In the following, we prove that $\mathbb{E} Z_{n} \rightarrow 0$.

From (3.2), we have

$$
\mathbb{E} Z_{n+1} \leq\left(1-2 \bar{\alpha}_{n} \beta_{n}-2 \bar{\beta}_{n}\right) \mathbb{E} Z_{n}+\frac{4}{(n+1)^{2}} .
$$

Since $0<\alpha_{n}, \beta_{n}<1$, it follows that

$$
1-2 \bar{\alpha}_{n} \beta_{n}-2 \bar{\beta}_{n}=1-2 \bar{\alpha}_{n} \beta_{n}-2\left(1-\beta_{n}\right)=2 \beta_{n}\left(1-\bar{\alpha}_{n}\right)-1=2 \alpha_{n} \beta_{n}-1 \leq \alpha_{n} \beta_{n},
$$

which, combined with (3.3), implies that

$$
\mathbb{E} Z_{n+1} \leq \alpha_{n} \beta_{n} \mathbb{E} Z_{n}+\frac{4}{(n+1)^{2}} .
$$

Note that $0<\alpha_{n} \beta_{n}<1, \prod_{i=1}^{n} \alpha_{i} \beta_{i}=s_{1} t_{1} / s_{n+1} t_{n+1} \rightarrow 0$ and $\sum_{n=1}^{\infty} 4 /(n+1)^{2}<\infty$. Hence, applying Lemma 3.1 below to (3.4) gives the desired conclusion, $\mathbb{E} Z_{n} \rightarrow 0$.

Lemma 3.1. Suppose that $\left\{x_{n}\right\}_{n \geq 1},\left\{a_{n}\right\}_{n \geq 1}$, and $\left\{b_{n}\right\}_{n \geq 1}$ are nonnegative real sequences satisfying $x_{n+1} \leq a_{n} x_{n}+b_{n}$, where $0<a_{n}<1$ for $n \geq 1$. If $\prod_{i=1}^{n} a_{i} \rightarrow 0$ and $\sum_{n=1}^{\infty} b_{n}<\infty$, then $x_{n} \rightarrow 0$.

Proof. First, note that $x_{n+1} \leq x_{n}+b_{n}$ since $0<a_{n}<1$. Thus,

$$
x_{n+1} \leq x_{1}+\sum_{i=1}^{n} b_{i} \leq x_{1}+\sum_{i=1}^{\infty} b_{i},
$$

which implies that $\left\{x_{n}\right\}_{n \geq 1}$ is uniformly bounded by a positive constant $M$.

Given $\varepsilon>0$, choose $n_{0}$ such that $\sum_{n=n_{0}}^{\infty} b_{i}<\varepsilon /(1+M)$, and then choose $n_{1}>n_{0}$ such that $\prod_{i=n_{0}}^{n} a_{i}<\varepsilon /(1+M)$ whenever $n>n_{1}$. Now, for each $n>n_{1}$, we have

$$
\begin{aligned}
x_{n+1} & \leq a_{n} x_{n}+b_{n} \\
& \leq a_{n} a_{n-1} x_{n-1}+b_{n-1}+b_{n} \\
& \vdots \\
& \leq\left(\prod_{i=n_{0}}^{n} a_{i}\right) x_{n_{0}}+\sum_{i=n_{0}}^{n} b_{i} \\
& \leq \frac{\varepsilon M}{1+M}+\frac{\varepsilon}{1+M} \\
& =\varepsilon .
\end{aligned}
$$

Hence, $x_{n} \rightarrow 0$, as required. 


\section{Acknowledgements}

We wish to thank the referee for an extremely careful reading of the manuscript and helpful suggestions. The first author also gratefully acknowledges support from the National Science Council of Taiwan under grant NSC 101-2118-M-110-001.

\section{References}

[1] Athreya, K. B. And Lahiri, S. N. (2006). Measure Theory and Probability Theory. Springer, New York.

[2] Bagchi, A. ANd PAL, A. K. (1985). Asymptotic normality in the generalized Pólya-Eggenberger urn model, with an application to computer data structures. SIAM J. Algebraic Discrete Methods 6, 394-405.

[3] Bernard, S. R., Sobel, M. and Uppuluri, V. R. R. (1981). On a two urn model of Pólya-type. Bull. Math. Biol. 43, 33-45.

[4] Chen, M.-R. and Wei, C.-Z. (2005). A new urn model. J. Appl. Prob. 42, 964-976.

[5] Eggenberger, F. And Pólya, G. (I923). Über die Statistik verketetter Vorgänge. Zeitschrift für Angewandte Mathematik und Mechanik. 3, 279-289.

[6] Gouet, R. (1989). A martingale approach to strong convergence in a generalized Pólya-Eggenberger urn model. Statis. Prob. Lett. 8, 225-228.

[7] Higueras, I., Moler, J., Plo, F. and San Miguel, M. (2003). Urn models and differential algebraic equations. J. Appl. Prob. 40, 401-412.

[8] Hill, B. M., Lane, D. and Sudderth, W. (1980). A strong law for some generalized urn processes. Ann. Prob. 8, 214-226.

[9] Johnson, N. L. And Kotz, S. (1977). Urn Models and Their Application, Wiley, New York.

[10] Kotz, S. And Balakrishnan, N. (1997). Advances in urn models during the past two decades. In Advances in Combinatorial Methods and Applications to Probability and Statistics, ed. N. Balakrishnan, Birkhäuser, Boston, pp. 203-257.

[11] Mahmoud, H. M. (2009). Pólya Urn Models, CRC Press, Boca Raton, FL.

[12] Martin, C. F. and Allen, L. J. S. (1995). Urn model simulations of a sexually transmitted disease epidemic. Appl. Math. Comput. 71, 179-199.

[13] Pemantle, R. (1990). A time-dependent version of Pólya's urn. J. Theoret. Prob. 3, 627-637.

[14] Renlund, H. (2010). Generalized Pólya urns via stochastic approximation. Preprint, available at http://arxiv.org/ abs/ 1002.3716 . 